\title{
Probabilistic load flow with wind production uncertainty using cumulants and Cornish-Fisher expansion
}

\author{
Julio Usaola \\ Department of Electrical Engineering, Universidad Carlos III de Madrid, Leganés, Madrid, Spain
}

\section{A R T I C L E I N F O}

\section{Article history:}

Received 26 September 2008

Accepted 17 February 2009

\section{Keywords:}

Probabilistic load flow

Wind generation

Cumulants

Cornish-Fisher expansion series

\begin{abstract}
A B S T R A C T
This paper proposes a method for probabilistic load flow in networks with wind generation, where the uncertainty of the production is non Gaussian. The method is based on the properties of the cumulants of the probability density functions (PDF) and the Cornish Fisher expansion, which is more suitable for non Gaussian PDF than other approaches, such as Gram Charlier series. The paper includes examples and comparisons between different methods proposed in literature.
\end{abstract}

\section{Introduction}

The great proliferation of wind energy in power networks has in creased the uncertainty of power system operation and manage ment. This uncertainty affects both the long and medium term system planning, and the day ahead operation. In both cases, to have an assessment of the values of the system variables under these uncertain conditions is important for the Transmission Sys tem Operator. This is why the importance of probabilistic tools for power system analysis is increasingly growing. For long term studies, to consider the uncertainty in power planning may lead to a less expensive network. For the daily operation, an adequate assessment of the system variables may lead to a better manage ment of congestions and other important advantages.

Among these tools, probabilistic power flow is one of the best known. To study all possible combinations of generation and load is impractical due to the great size of real networks, and this is why analytical methods should be used to adequately assess the vari ability of the grid magnitudes. From the first proposals in the sev enties [1,2], a great deal of literature can be found about this subject. In these references, it was mainly the uncertainty of the load what was considered.

The most straightforward method of solving this problem is Monte Carlo simulation. This technique involves repeated simu lation with values obtained from the PDF of the random vari able considered. But for an adequate representation, a great deal of simulations must be considered in real systems. This

E-mail address: jusaola@ing.uc3m.es makes this approach unpractical. One of the alternatives to this is the convolution of the PDF of the random variables involved, when they are independent of each other, and linearly related. Although this reduces the computational burden, it is still a for midable task to obtain the PDF of a single line when several random power injections are considered. Fast Fourier Transform (FFT) techniques were proposed to reduce the computational burden [3], but this method is linked to the convolution tech nique, and does not solve the problem efficiently. Recently, the use of cumulants and the approximation of resultant PDF by orthogonal series (Gram Charlier expansion series) have been proposed [4]. It has interesting properties, and is compu tationally inexpensive.

Probabilistic load flow has been applied mostly to consider the uncertainty of load. The uncertainty of load is not very high, and it is frequently modeled using Gaussian probabilistic density functions. Wind energy proliferation, however, rends this ap proach insufficient, since the variability of wind power produc tion is much higher, and usually the PDF are not Gaussian. Long term planning studies must consider PDF based on Weibull distri butions, while short term operation analysis need to use PDF whose properties are still under study. Ref. [5] proposes the use of FFT and convolution in distribution networks, and makes esti mates, under simplified conditions, of the PDF for short term wind power prediction. For large transmission networks it seems that the approach based on cumulants is very appropriate due to the low computational requirements. However, for non Gaussian PDF, Gram Charlier expansion series have serious convergence problems, and alternative tools have to be proposed. In this paper, an approach using Cornish Fisher expansion is presented. This 
method has better convergence properties, without more compu tational burden.

The method is applied to next day operation of systems with great wind penetration. Usually, the system operators of these net works use the output of a short term wind power prediction pro gram to forecast the next day grid constraints and congestions. However, these predictions have a lower accuracy than load fore casts, and their uncertainty should be also taken into account, for instance for the available interconnection capacity between sys tems. Therefore, a tool able to assess the probability of surpassing the allowed line capacity is very practical for an adequate operation.

The paper is structured as follows: first, a short view of short term wind power prediction, and the uncertainty associated to its output, is given. Then, the theoretical foundations of the Cor nish Fisher expansion series and the necessary definitions are introduced. The method is applied to a test grid, and its results are compared to those obtained using Gram Charlier expansion series. A summary with the main conclusions ends the paper.

\section{Short term wind power prediction: uncertainty}

\subsection{Short term wind power prediction}

Short term wind power prediction programs are tools that pro vide an estimate of the future power production of a wind farm, or a group of wind farms, in the next hours. For this purpose, they use meteorological forecasts coming from a Numerical Weather Pre diction (NWP) tool, and sometimes real time SCADA data from the wind farms, namely, wind power production and other values, such as measured wind speed. Data of the wind farms, such as rated power, type and availability of wind turbines, etc. are also necessary. The output of these programs is the hourly average wind farm production for the next hours. Typically, predictions are issued for the next 48 hours, but longer time horizons are pos sible, sometimes at the price of a poorer accuracy.

These prediction tools are less accurate than load prediction programs and their accuracy decreases with the time horizon. An example of this accuracy for a typical wind farm is given in Fig. 1, where the output from the prediction program SIPREOLICO [6] is compared to persistence. Persistence is a prediction method that consists of assuming that the future prediction, for the entire time horizon considered, is the current production of the wind farm.

The figure represents the Normalized Mean Average Error, de fined as

$\operatorname{NMAE}(k) \quad \frac{1}{P_{n}} \frac{\sum_{t=1}^{N}|e(t+k / t)|}{N}$

where

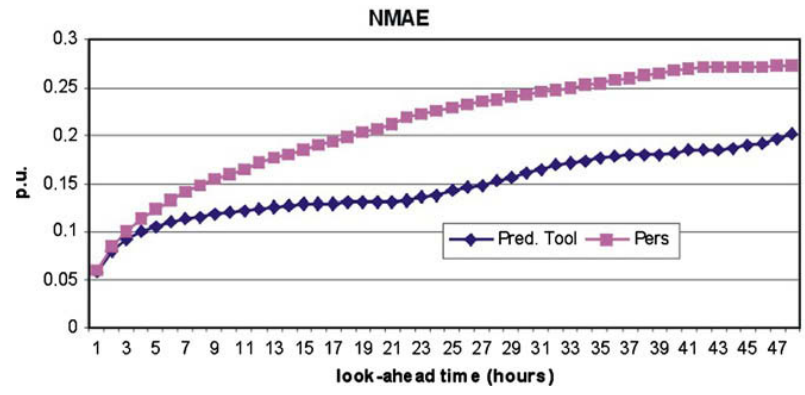

Fig. 1. NMAE of a typical wind farm, for a prediction tool and persistence. $e(t+k / t) \quad p(t+k) \quad \hat{p}(t+k / t)$

And $p(t+k)$ is the production of the wind farm at time $(t+k)$, while $\hat{p}(t+k / t)$ is the power predicted at time $t$ for time $(t+k) . P_{n}$ is the nominal power of the wind farm, and $N$ is the number of predictions examined along the considered time. It can be seen that the wind power prediction accuracy allows for much uncertainty, and that the actual value may differ widely from the predicted one.

\subsection{Uncertainty of short term wind power prediction}

The predictions provided by a short term wind power predic tion program are uncertain, and this uncertainty must be modeled for an adequate assessment of these predictions.

The uncertainty, and hence its probability density function, changes with the range of the wind farm power output, since this value is bounded between zero and the rated power. Besides, the power curve of a wind turbine or wind farm is nonlinear. If we as sume that the wind speed forecasts have Gaussian uncertainty, then the probability density functions of the power predictions will not be Gaussian. The shape of these probability density functions is also affected by the time lag elapsed between the prediction and the operation times. A sample of an heuristical PDF of the uncer tainty of short power prediction is given in Fig. 2. This function shows the uncertainty of a wind power prediction made with a time horizon of 7 hours when the forecasted power was 0.2 p.u.

It is not within the purposes of this paper to propose a model for this uncertainty and a reasonable assumption will be used as an approximation. Due to the bounded nature of the power produced by a wind far, a Beta PDF will be used, as proposed in [7]. Heuristic PDF, as shown in [8] supports this assumption, although this is still an open field for research. In our case, the mean of the distribution will be the predicted power at the time of interest, while the stan dard deviation $\sigma$ will depend on the level of power injected, with respect to the wind farm rated power. This dependence has been obtained heuristically for some wind farms, and the results are shown in Fig. 3, where the value of standard deviation is normal ized to the rated power of the wind farm. Although there are wide variations, an approximation by a quadratic curve (shown in the picture) may provide realistic results.

All these values have been obtained from real production of three months of a wind farm whose rated power has been normal ized to 1 . Predictions have been made with the prediction tool SIP REOLICO [6].

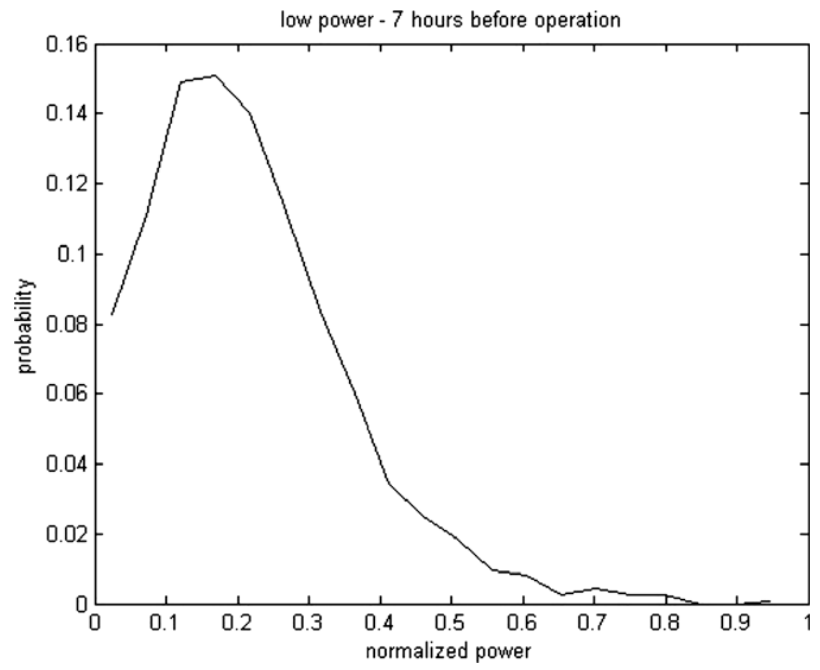

Fig. 2. Sample PDF of the uncertainty of wind power prediction. 


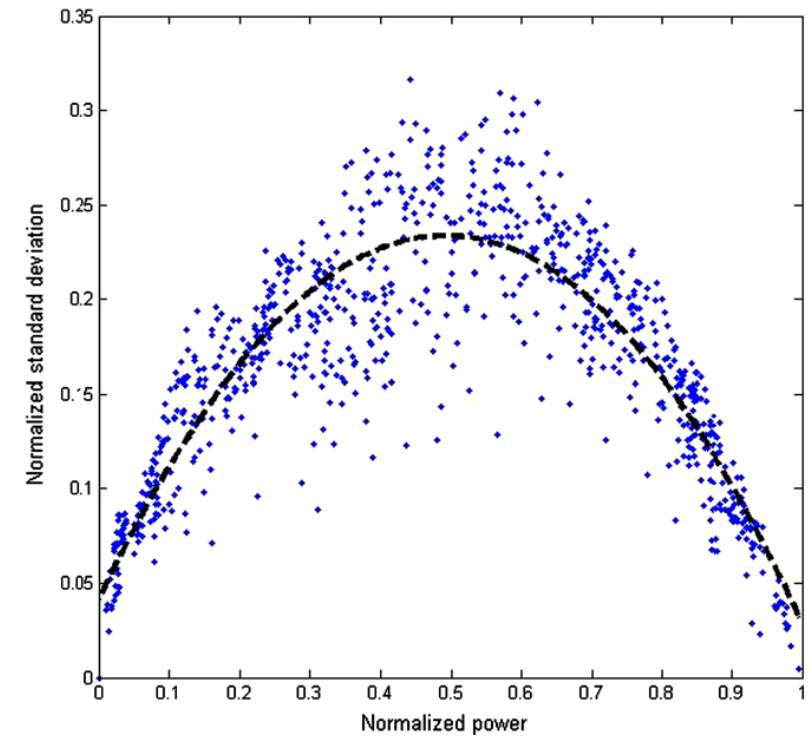

Fig. 3. Relation between standard deviation and mean for the uncertainty of predictions.

\section{Theoretical background}

A short introduction of probability theory is given here for a better understanding of the method. For more details, Refs. [9] or [12] can be consulted.

\subsection{Moments and cumulants}

For a random variable $x$ with probability density function (PDF) $f(x)$, the cumulative density function (CDF) $F(x)$, is defined as

$F(x) \quad \int_{\infty}^{\infty} f(x) d x$

For a given PDF, the moment of order $n$, where $n$ is an integer, is defined as:

$m_{n} \quad E\left[x^{n}\right] \quad \int_{\infty}^{\infty} x^{n} f(x) d x$

$m_{1} \quad \eta$ is the mean of the random variable.

Central moments, or moments about the mean are defined as

$\left.\mu_{n} \quad E\left[\begin{array}{ll}x & \eta\end{array}\right)^{n}\right] \quad \int_{\infty}^{\infty}\left(\begin{array}{ll}x & \eta\end{array}\right)^{n} f(x) d x$

$\mu_{2} \quad \sigma^{2}$ is the variance of the random variable.

The moment generating function, $\phi(s)$, associated to a random variable $x$ is defined as

$\phi(s) \quad E\left[e^{s x}\right] \quad \int_{\infty}^{\infty} e^{s x} f(x) d x$

It can be easily shown (see [9]) that

$\phi^{(n)}(0) \quad E\left[x^{n}\right] \quad m_{n}$

where $(n)$ indicates the $n$ derivative. Hence, $\phi(s)$ could be expanded as a MacLaurin series as:

$\phi(s) \quad \sum_{n}^{\infty} \frac{1}{n !} \phi^{(n)}(0) s^{n} \quad \sum_{n 0}^{\infty} \frac{m_{n}}{n !} s^{n}$

The cumulant generating function, $\psi(s)$, is defined as:

$\psi(s) \quad \ln \phi(s)$
And the cumulants of order $r, \kappa_{r}$, are finally defined as

$\kappa_{r} \quad \frac{d^{n} \psi(0)}{d s^{n}}$

From the series expansions of moment and cumulant generat ing functions, it can be written the following relation between mo ments and cumulants:

$\sum_{n}^{\infty} \frac{m_{n}}{n !} s^{n} \quad \exp \left\{\sum_{r 0}^{\infty} \frac{\kappa_{r}}{r !} s^{r}\right\}$

If the exponential is expanded in its Taylor series, and the terms of powers of $s$ are equaled, the relations between moments and cumulants can be found as (12).

$\kappa_{r+1} \quad m_{r+1} \sum_{j=1}^{r}\left(\begin{array}{l}r \\ j\end{array}\right) m_{j} \kappa_{r}{ }_{j+1}$

A similar expression between cumulants and central moments can be written [10].

\subsection{Properties of cumulants}

Let $x_{1}$ and $x_{2}$ be two independent variables, with $\operatorname{PDF} f_{x_{1}}\left(x_{1}\right)$ and $f_{x_{2}}\left(x_{2}\right)$. Then, the PDF of the random variable $z$, where $z \quad x_{1}+x_{2}$ is given by

$f_{z}(z) \quad f_{x_{1}}\left(x_{1}\right) * f_{x_{2}}\left(x_{2}\right)$

where $*$ indicates convolution. Then, it can be easily demonstrated that the cumulants of order $r$ of the random variable $z, \kappa_{z, r}$, are given by

$\kappa_{z, r} \quad \kappa_{x_{1}, r}+\kappa_{x_{2}, r}$

In general, when $z$ is a linear combination of $J$ random variables, $x_{1}, \ldots, x_{j}, z \quad \sum_{j 1}^{J} a_{j} x_{j}$, then

$\kappa_{z, r} \quad \sum_{j=1}^{J} a_{j}^{r} \kappa_{x_{j}, j}$

A generalization of Eq. (15) that can be applied to dependent variables can be found in [10].

\subsection{Gram Charlier series expansion}

In theory, it is possible to obtain the PDF, or the CDF of a random variable, if its moments, or cumulants, are known, at least for some families of CDF. In practice, however, this is a difficult problem, still open, and different proposals have been made to solve it, only with partial success. One of these proposals is the Gram Charlier series.

Let consider the series expansion of a $\operatorname{CDF} F(x)$ with mean $\eta \quad 0$ and $\sigma \quad 1$ in terms of a base function $\sigma(x)$, where $\sigma(x)$ is a function $\mathrm{N}(0,1)$. This expansion can be written as:

$F(x) \quad \sum c_{j} \Phi^{(j)}(x)$

where $\sigma^{(j)}(x)$ is the $j$ th derivate of $\sigma(x)$. This equation can be written in terms of the Tchebycheff Hermite polynomials (see Appendix A) as

$F(x) \quad \sum_{j 0}^{\infty} c_{j} H_{j} \Phi(x)$

Multiplying by $H_{r}(x)$ and integrating from $\infty$ to $\infty$, we have in virtue of the orthogonal relationship between Tchebycheff Her mite polynomials 
$c_{r} \frac{1}{r !} \int_{\infty}^{\infty} F(x) H_{r}(x) d x$

From these equations, the values of $c_{r}$, may be obtained as functions of the central moments. The first four terms are:

$$
\begin{aligned}
& \begin{array}{llll}
c_{1} & 1 & c_{2} & 0
\end{array} \\
& c_{3} \quad \frac{1}{3 !} \mu_{3} \quad c_{4} \quad \frac{1}{4 !}\left(\begin{array}{ll}
\mu_{4} & 3
\end{array}\right)
\end{aligned}
$$

It must be remarked that central moments can be easily ob tained from cumulants [12].

Eq. (17) is the Gram Charlier series of Type A. It can be demon strated that this infinite series converge if the integral

$\int_{\infty}^{\infty} e^{\frac{x^{2}}{4}} f(x) d x$

converges, and if $f(x)$ tends to zero as $|x|$ tends to infinity, where $f(x)$ is the PDF, i.e. the derivative of $F(x)$. This limits the valid dis tributions only to a reduced number of the most common distri butions. From the statistical viewpoint, however, the important question is not whether an infinite series can represent a fre quency function, but whether a finite number of terms can do so to a satisfactory approximation. It is possible that even when the infinite series diverges its first few terms will give an approx imation of an asymptotic character. Actually, the series in the Charlier form may behave irregularly in the sense that the sum of $k$ terms may give a worse fit than the sum of $\left(\begin{array}{ll}k & 1\end{array}\right)$ terms. In many statistical inquiries we are more interested in the tails of a distribution than its behavior in the neighborhood of the mode, and it is here that the Gram Charlier series appears partic ularly inadequate [11 13].

\subsection{Cornish Fisher expansion}

Cornish Fisher expansion is related to the Gram Charlier series [14]. This approach provides an approximation of a quantile $\alpha$ of a cumulative distribution function $F(x)$ in terms of the quantile of a normal $\mathrm{N}(0,1)$ distribution $\sigma$ and the cumulants of $F(x)$. The theo retical deduction of this expansion is quite complex, and can be found in [12] or [14], for instance.

Using the first five cumulants, the expansion is given by (19).

$$
\begin{aligned}
& x(\alpha) \approx \xi(\alpha)+\frac{1}{6}\left(\xi^{2}(\alpha) \quad 1\right) \kappa_{3}+\frac{1}{24}\left(\xi^{3}(\alpha) \quad 3 \xi(\alpha)\right) \kappa_{4} \\
& \frac{1}{36}\left(2 \xi^{3}(\alpha) \quad 5 \xi(\alpha)\right) \kappa_{3}^{2}+\frac{1}{120}\left(\xi^{4}(\alpha) \quad 6 \xi^{2}(\alpha)+3\right) \kappa_{5} \\
& \frac{1}{24}\left(\xi^{4}(\alpha) \quad 5 \xi^{2}(\alpha)\right) \kappa_{2} \kappa_{3}+\frac{1}{324}\left(12 \xi^{4}(\alpha) \quad 53 \xi^{2}(\alpha)\right) \kappa_{3}^{3}(19)
\end{aligned}
$$

where $x(\alpha) \quad F^{1}(\alpha), \xi(\alpha) \quad \sigma^{1}(\alpha)$ and $\kappa_{r}$ is the cumulant of order $r$ of the cumulative distribution function $F$.

Although the convergence properties of Cornish Fisher series are difficult to demonstrate [15], and are somehow related to Gram Charlier series, their behavior for non Gaussians PDF is bet ter than the latter, as will be shown below.

\section{Probabilistic load flow formulation}

\subsection{Probabilistic load flow}

Probabilistic power flow is a tool that provides the probability of a system variable taking a value. These variables may be node voltages, power through lines, or any other. The aim of this pro gram is to estimate the risk of line overloading and congestion for the next hours. For this purpose the DC load flow equations provide a good estimate of the power flows. Fig. 4 shows the rel ative error in \% of power flows in lines between AC and DC solu

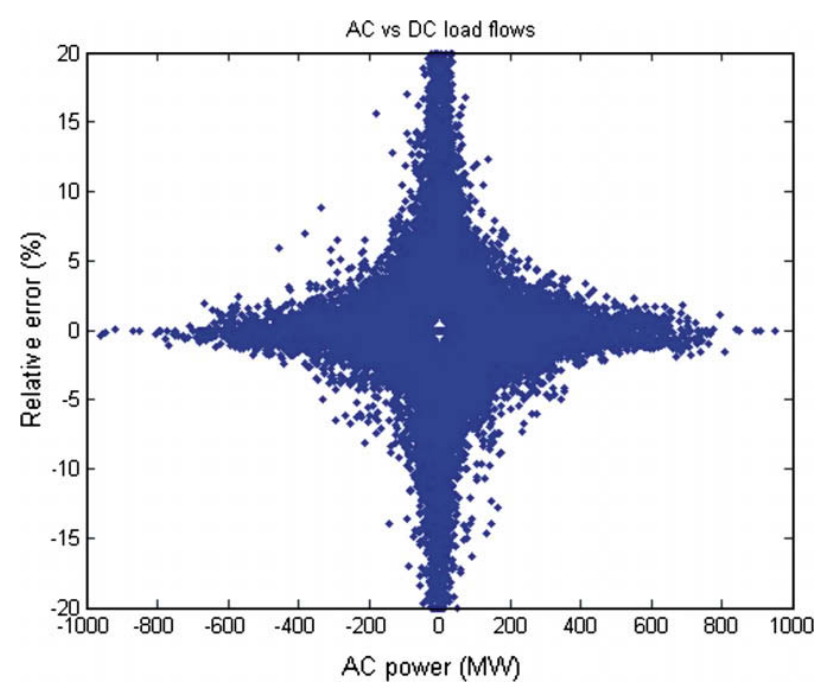

Fig. 4. Comparison between $A C$ and DC load flows in the Spanish peninsular transmission grid.

tion. This has been calculated for all the lines in the transmission network of the Peninsular Spanish power system, for 72 repre sentative cases along year 2004. The minimum power limit of the lines in the studied case is around $220 \mathrm{MW}$. Beyond this power, the error is less than $5 \%$ between DC and AC power flows.

Therefore, for large powers (with risk of overload, or conges tion) the errors are small enough, so DC load flow can be consid ered as an adequate approximation, at least as a first approach.

The well known DC load flow equations are:

$\mathbf{B}^{\prime} \boldsymbol{\delta} \quad \mathbf{P}$

\section{$\mathbf{P}_{f} \quad \mathbf{X}^{1} \mathbf{T}^{t} \boldsymbol{\delta} \quad \mathbf{X}^{1} \mathbf{T}^{t} \mathbf{B}^{\prime 1} \mathbf{P} \quad \mathbf{A} \quad \mathbf{P}$}

where $\mathbf{P}_{f}$ is the vector of power flows through lines, $\mathbf{P}$ is the vec tor of net nodal power injections, $\mathbf{B}^{\prime}$ is the susceptance matrix whose terms are $B_{i j} \quad 1 / X_{i j}$, and $B_{i i} \quad \sum_{i \neq j} 1 / X_{i j}, \delta$ is the vector of nodal voltage angles, $\mathbf{X}^{1}$ is a diagonal matrix whose terms are the inverse of the branch reactances, $\mathbf{T}$ is the branch node incidence matrix, and $\mathbf{A} \mathbf{X}^{1} \mathbf{T}^{t} \mathbf{B}^{\prime}{ }^{1}$ is the coefficient matrix that relates the line power flows to the nodal power injections. This establishes the linear relation between power flows and nodal power injections.

These coefficients are obtained assuming that the load or gener ation changes are compensated in the slack node. A generalization of this expression may be made if it is considered that the changes in the wind power production or load will be compensated by sev eral power plants, instead of only the slack node. This consider ation is reasonable in situations where the changes in generation may be high as it happens with wind energy, where expected vari ations from the expected production are usually high. Under these assumptions, the linear relation between branch flows and injected power is given by the coefficient matrix $\mathbf{A}^{\prime}$, whose terms are given in Eq. (21)

$$
\begin{array}{lll}
\mathbf{P}_{f} & \mathbf{A}^{\prime} & \mathbf{P} \\
a_{i j}^{\prime} & a_{i j} & \sum_{r} k_{j r} a_{i r}
\end{array}
$$

where $a_{i j}$ is th term $(i, j)$ of the coefficient matrix $\mathbf{A}, k_{j r}$ is the part of power injection in node $j$ that the regulating generator $r$ assumes, as defined previously, for example $\left(k_{j r} \quad 1 / R\right) . R$ is the number of gen erators that compensate the injection in node $j$. 


\subsection{Computational procedure}

The proposed method begins from a deterministic evaluation of line flows, using wind power predictions. Then, the probabilistic load flow follows, which provides the CDF of the lines of interest. It must be remarked that the deterministic prediction is the ex pected value of the predicted power.

Hence, the following steps should be followed to find the CDF of the line flows.

1. Solve a DC load flow with the expected value of the wind power injected to the system. This gives the mean (expected value) of line power flows.

2. Calculate the moments and cumulants of the CDF of the wind power injections, using (4) and (12).

3. Use Eq. (15) to find the cumulants of the random variables of the power flows through the lines of interest. Coefficients $a_{i j}$ are obtained from Eq. (21).

4. Use the Cornish Fisher expansion (19) to find the value of the $\mathrm{CDF}$ of the power in the lines of interest.

\section{Case study}

In order to verify the better convergence properties of this method, its results are compared with Monte Carlo simulation and Gram Charlier expansion series. The method has been applied to the IEEE RTS96 system [16], modified to include two wind farms. The grid, with the changes from the original system is shown in Fig. 5. These wind farms may represent, in reality, groups of wind farms connected to the transmission network. In the fig ure, both the rated power $\left(P_{n}\right)$ and the predicted power $(P)$ for a certain time are written.

The lines of interest, where the PDF of the power flows are to be found are the lines 1716 and 1722 , marked in the same figure.

Although the power predicted is 300 and $200 \mathrm{MW}$, these values are the expected values of the PDF of the injected power. Since the

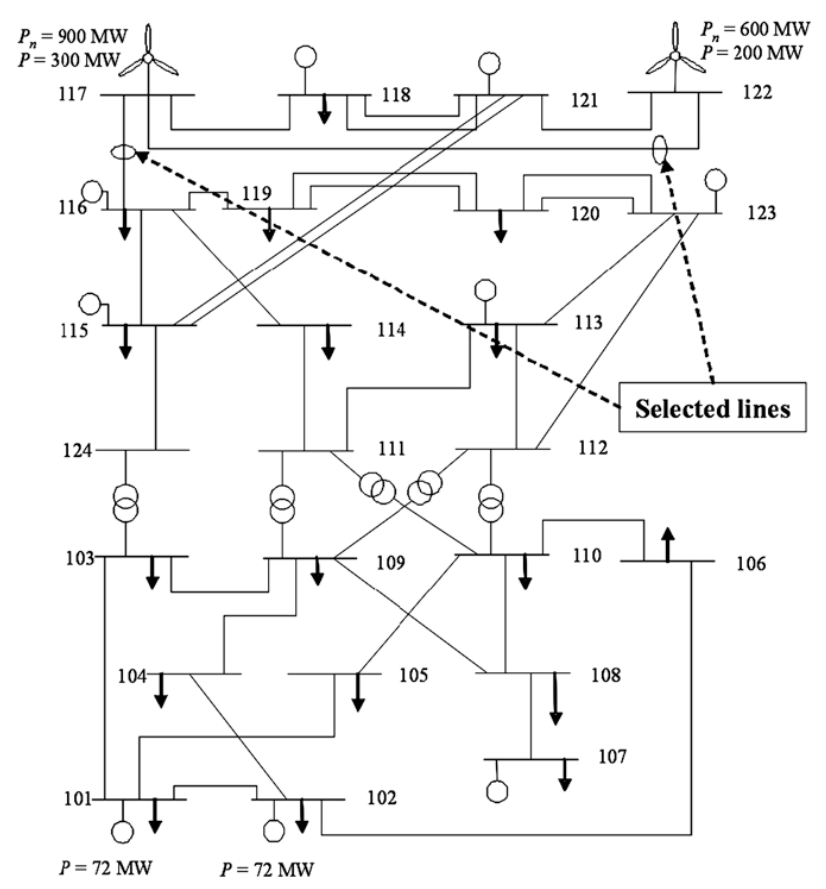

Fig. 5. IEEE-RTS96 system with wind farms connected.

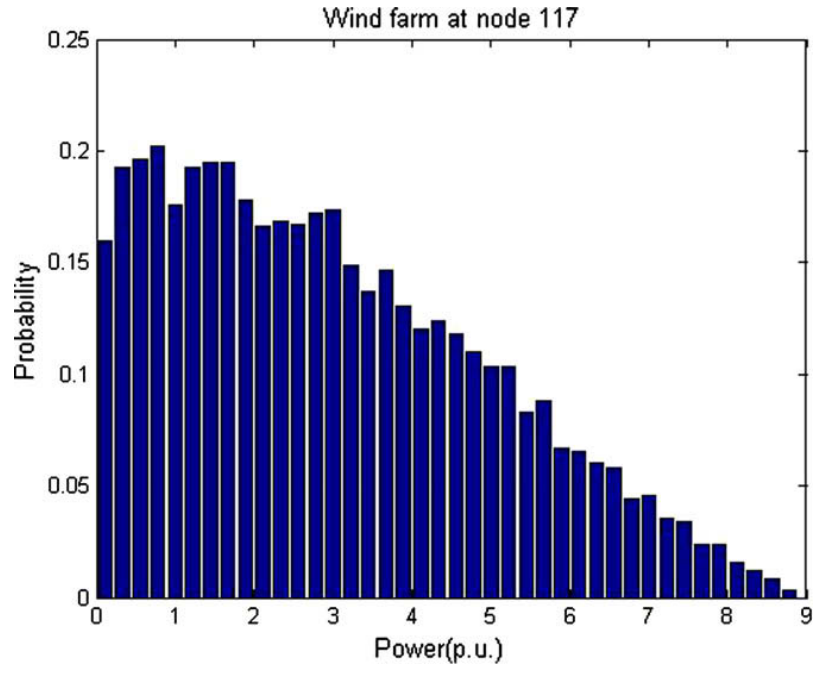

Fig. 6. PDF of the power injected by the wind farm connected to node 117 .

power ranges of the predictions are low with respect to the wind farm nominal values, the PDF of the injected powers have been modeled as beta functions, as previously described. The frequency distribution for a Monte Carlo sampling of 10,000 samples, are shown in Figs. 6 and 7. Base power is $100 \mathrm{MW}$. The two random variables are considered independent.

\section{Results}

For this case, the results obtained using the Gram Charlier ser ies and the Cornish Fisher expansion are compared to the results from Monte Carlo simulation. The purpose of this paper is to prove the better convergence properties of the Cornish Fisher expansion, when non Gaussian functions are involved. Computation times be tween Gram Charlier and Cornish Fisher are equivalent, and it has been demonstrated in other publications, as [4], the smaller com putation times of Gram Charlier series method compared to Monte Carlo method, so a comparison of computation times will not be made here.

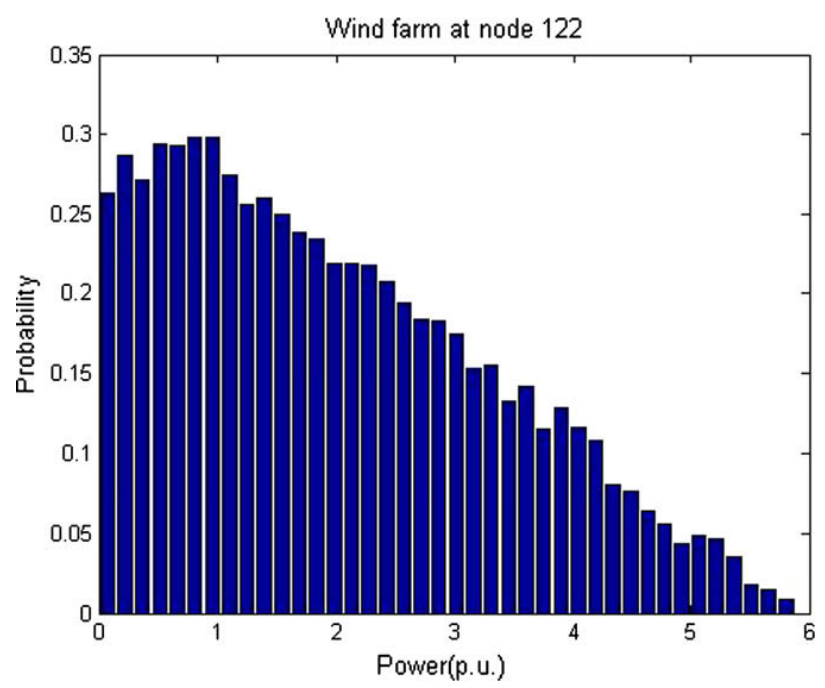

Fig. 7. PDF of the power injected by the wind farm connected to node 122 . 
Table 1

Values of $\varepsilon_{n}$ for the power flows in the branches of the grid considered.

\begin{tabular}{llllll}
\hline Moment & 1 & 2 & 3 & 4 & 5 \\
\hline Error (\%) & 0.5230 & 0.1535 & 1.8661 & 0.7126 & 2.0679 \\
\hline
\end{tabular}

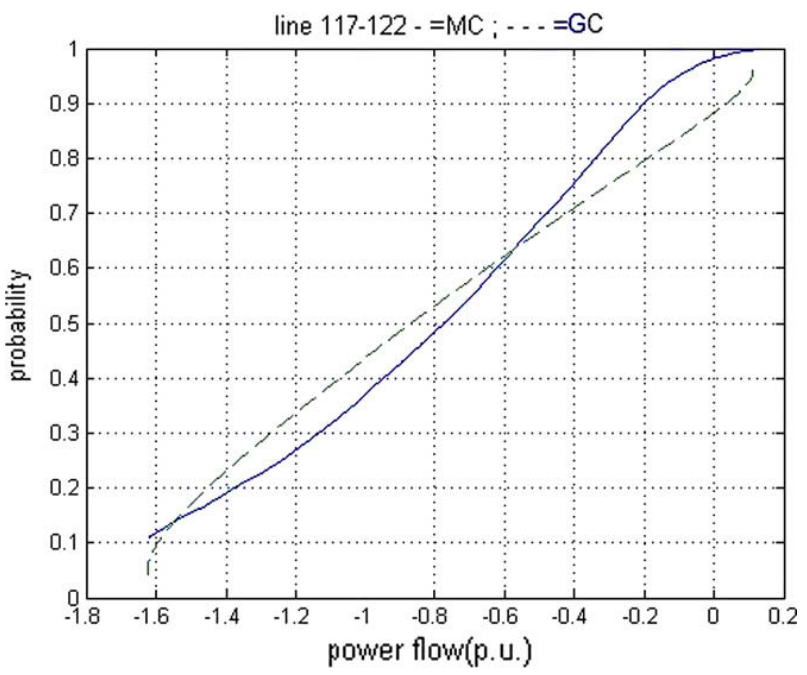

Fig. 8. CDF obtained with Monte Carlo simulation and with Gram-Charlier expansion series (dotted line). Line 117-122.

The number of Monte Carlo samples is 10,000 . With this num ber of samples there is a 95\% probability that the greatest error in the mean of the power in the considered branches is smaller than $3.95 \%$.

The method obtains a very good approximation to the moments of power flows through lines. Let the error in the approximation of the central moment of order $n$ be defined in the following way:

$\varepsilon_{n} \quad \frac{1}{N_{B}} \sum_{j=1}^{N_{B}} \frac{\left|\mu_{n, j}^{a n} \quad \mu_{n, j}^{M C}\right|}{\left|\mu_{n, j}^{M C}\right|} 100$

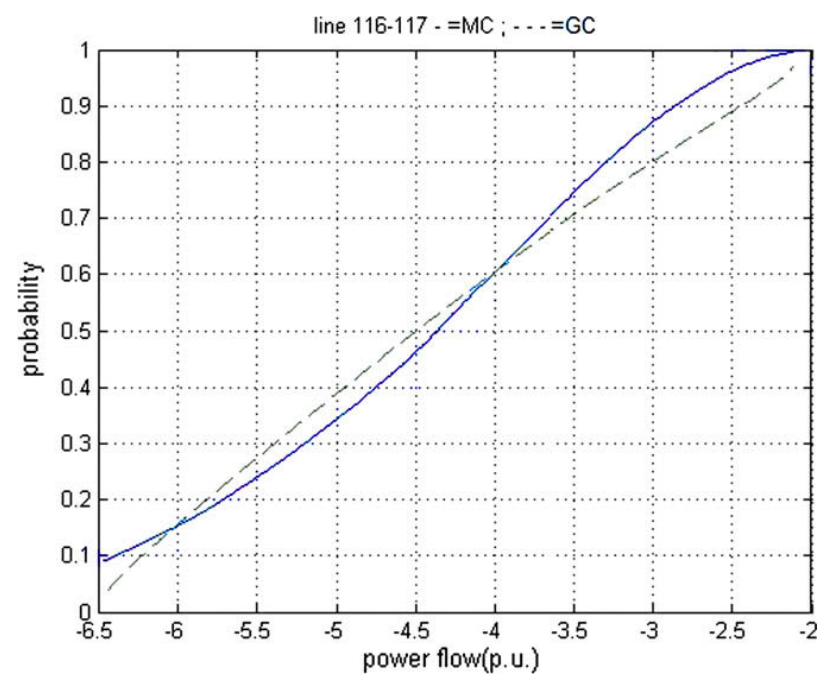

Fig. 9. CDF obtained with Monte Carlo simulation and with Gram-Charlier expansion series (dotted line). Line 116-117.

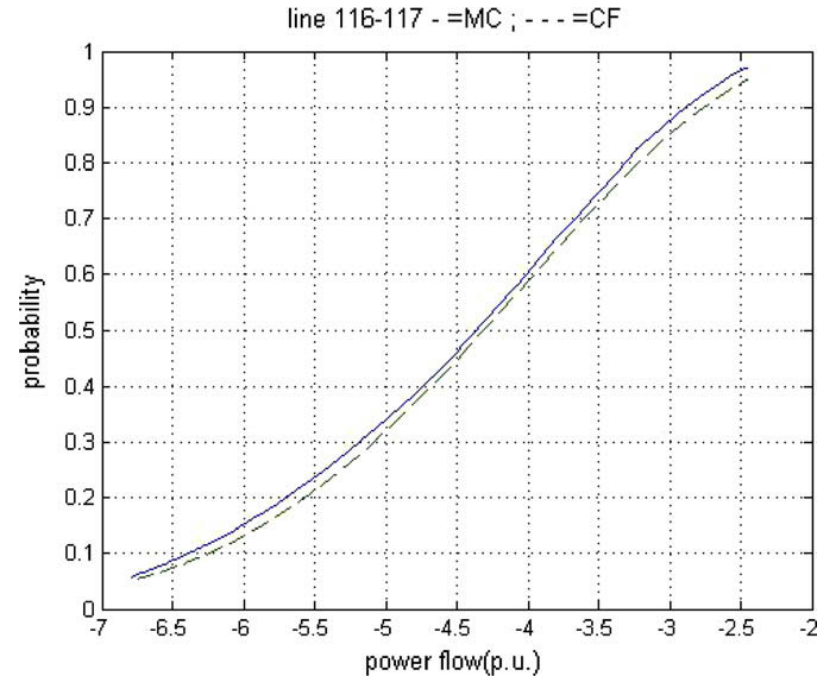

Fig. 10. CDF obtained with Monte Carlo simulation and with Cornish Fisher expansion (dotted line). Line 116-117.

where $\mu_{n, j}^{a n}$ is the central moment of order $n$ of branch $j$ found ana lytically, while $\mu_{n j}^{M C}$ is the same moment obtained by Monte Carlo method. $N_{B}$ is the number of branches in the grid.

Then, the value of this error for all the branches in the grid con sidered is given in Table 1.

Figs. 8 and 9 show the CDF of the lines of interest, using Gram Charlier expansion series, in comparison with the results using Monte Carlo. It can be seen that the behavior of the Gram Charlier is poor for both lines.

Figs. 10 and 11 show the CDF for both lines obtained using the Cornish Fisher expansion, in comparison with the result obtained

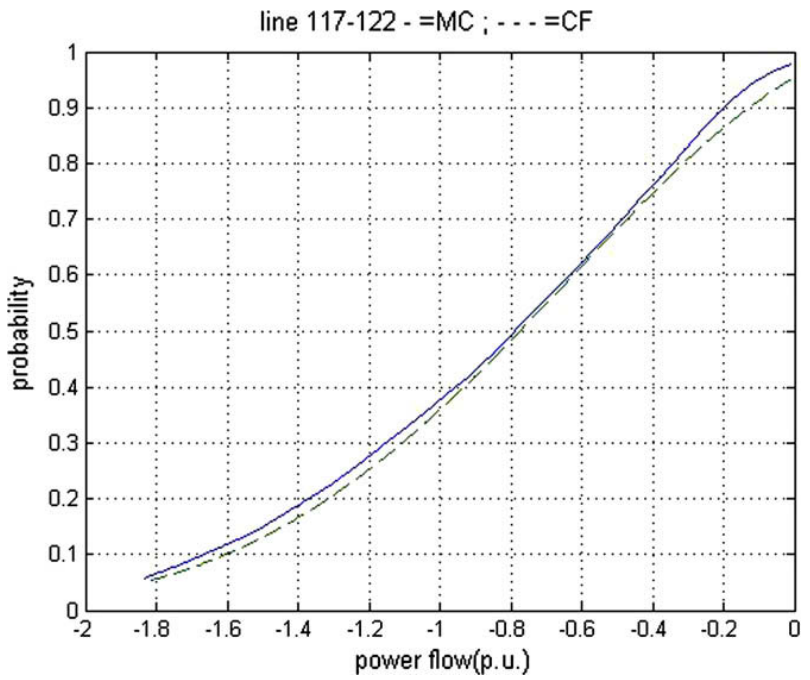

Fig. 11. CDF obtained with Monte Carlo simulation and with Cornish Fisher expansion (dotted line). Line 117-122.

Table 2

Comparison of the results for the $90 \%$ values of the lines of interest.

\begin{tabular}{lll}
\hline Lines & Absolute error (p.u.) & Relative error (\%) \\
\hline $117-122$ & 0.1283 & 1.9974 \\
$116-117$ & 0.0655 & 3.8884 \\
\hline
\end{tabular}




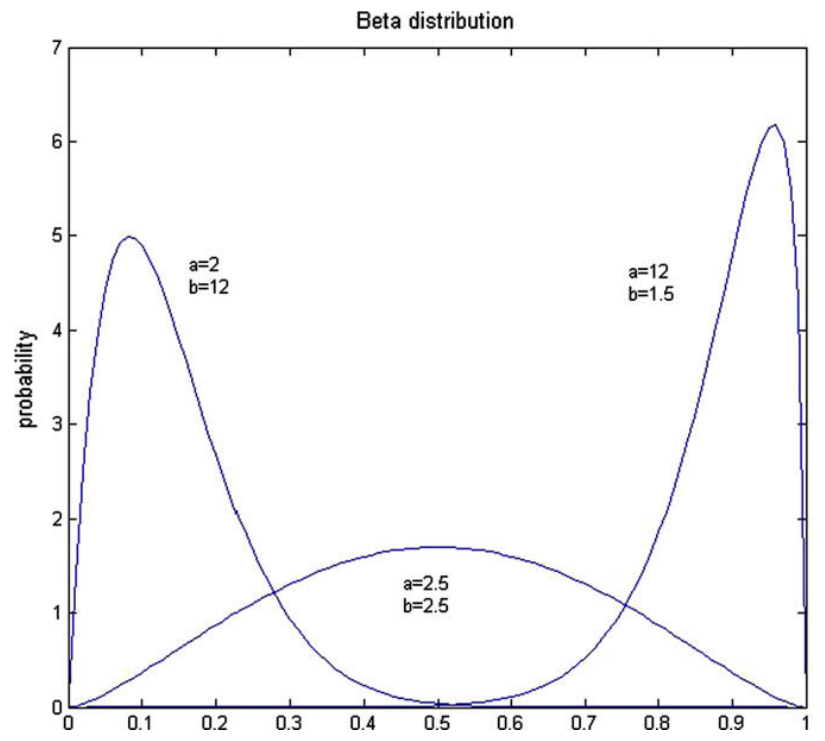

Fig. 12. Beta distribution for different values of parameters $a$ and $b$.

using Monte Carlo. It can be observed that the fitting in this case is very good.

Another numerical comparison given here is the difference be tween the values given by Monte Carlo method and Cornish Fisher expansion for a quantile of $90 \%$. This is shown in Table 2 .

The accuracy of these results allows to consider Cornish Fisher expansion more adequate for the problem conditions (non Gauss ian PDF of the wind power uncertainties), instead of the Gram Charlier expansion series. More examples have been run with identical result. Only when the PDF of the injection power are Gaussian, are the results comparable.

\section{Conclusion}

The operation of power systems with high wind power penetra tion must consider the uncertainty of short term wind power pre diction, and therefore new analysis tools must be used to deal with it. System Operators usually work with expected values for the in jected power, but due to the relatively low accuracy of the predic tions, actual values may differ widely from those expected and the system variables may be also very different from those planned.

In relation with this problem, it has been shown that the Cor nish Fisher expansion represents an interesting method for per forming probabilistic load flows in networks with wind power, where the PDF of the power injections are non Gaussian. Under these conditions, other methods like Gram Charlier series are less adequate because of their worse convergence properties. Although the convergence of Cornish Fisher series have not been obtained theoretically, empirical results show that they fit better the non Gaussian nature of the PDF involved.

The next developments of this work will be centered on the modeling of prediction uncertainty, the consideration of depen dence between random variables and other ways of building the resulting PDF from the moments.

\section{Acknowledgements}

This research has been carried out within the research projects Anemos Plus (6th FP European Project. Reference 38692) and IE MEL, Research Project of the Spanish Ministry of Education (Refer ence ENE2006 05192/ALT). Most of this work has been made during a sabbatical leave from the Universidad Carlos III de Madrid in the Ecole Superieure d'Electricité (Supélec), France. The stay has been also financed by the Spanish Ministry of Education within the program "Estancias de profesores e investigadores españoles en centros de enseñanza superior e investigacion extranjeros" (Refer ence PR2007 0032).

\section{Appendix A. Tchebycheff-Hermite polynomials}

A distribution $\varphi(x)$ that is $\mathrm{N}(0,1)$ can be written as

$\varphi(x) \frac{1}{\sqrt{ } 2 \pi} e^{\frac{1}{2} x^{2}}$

If we call $D \quad \frac{d}{d x}$, the successive derivatives with respect to $x$ are:

$D \varphi(x) \quad x \varphi(x)$

$D^{2} \varphi(x) \quad\left(\begin{array}{ll}x^{2} & 1\end{array}\right) \varphi(x)$

$D^{3} \varphi(x) \quad\left(3 x \quad x^{3}\right) \varphi(x)$

The result will be a polynomial in $x$ multiplied by $\varphi(x)$. We then define the Tchebycheff Hermite polynomial $H_{r}(x)$ by the identity

$(D)^{r} \varphi(x) \quad H_{r}(x) \varphi(x)$

$H_{r}(x)$ is of degree $r$ in $x$ and the coefficient of $x^{r}$ is unity. By conven tion $H_{0} \quad 1$, the following recurrence equation gives the value for $r>0$.

$H_{r}(x) \quad x H_{r_{1}}(x) \quad(r \quad 1) H_{r_{2}}(x)$

The polynomials have an important orthogonality property, namely, that

$$
\begin{aligned}
& \int_{\infty}^{\infty} H_{m}(x) H_{n}(x) \varphi(x) d x \quad 0 \quad m \neq n \\
& n \text { ! } m \quad n
\end{aligned}
$$

\section{Appendix B. Beta distribution}

The analytical expression of beta probability density function is

$f(x ; a, b) \quad \frac{1}{B(a, b)} x^{a}{ }^{1}(1 \quad x)^{b} 1$

where $B(a, b)$ is the beta function, and $a$ and $b$ are parameters re lated to the mean, $\eta$, and the variance, $\sigma^{2}$, in the following way:

$\eta \quad \frac{a}{a+b} \quad \sigma^{2} \quad \frac{a b}{(a+b)^{2}(a+b+1)}$

The beta distribution has been represented in Fig. 12 .

\section{References}

[1] Borkowska B. Probabilistic load flow. IEEE Trans Power App Syst 1974;PAS 93(May):752-9.

[2] Dopazo JF. Stochastic load flow. IEEE Trans Power App Syst 1975;PAS94(March):299-309.

[3] Allan RN, Da Silva AML, Burchett RC. Evaluation methods and accuracy in probabilistic load flow solutions. IEEE Trans Power App Syst 1981;PAS100(May):2539-46.

[4] Zhang P, Lee T. Probabilistic load flow computation using the method of combined cumulants and Gram-Charlier expansion. IEEE Trans Power Sys 2004;PWRS-19(February):676-82.

[5] Hatziargyriou ND, Karakatsanis TS, Papadopoulos M. Probabilistic load flow in distribution systems containing dispersed wind power generation. IEEE Trans Power Sys 1993;PWRS-8(February):159-65.

[6] González G, Díaz-Guerra B, Soto F, López S, Sánchez I, Usaola J, et al. SIPREÓLICO - wind power prediction tool for the Spanish peninsular power system. In: Proceedings of the CIGRÉ 40th general session and exhibition, París, France; August 2004

[7] Bludszuweit H, Domínguez-Navarro JA, Llombart A. Statistical analysis of wind power forecast error. IEEE Trans Power Sys 2008;PWRS-23(3). 
[8] Usaola, J, Angarita J. Bidding wind energy under uncertainty. In: Proceedings of the 2007 ICCEP, Capri, Italy; May 2007

[9] Papoulis A, Pillai SU. Probability, random variables and stochastic processes. McGraw-Hill; 2002.

10] McCullagh P. Tensor methods in statistics. London: Chapman and Hall; 1987

[11] Cramer H. Numerical methods of statistics. Princeton, NJ: Princeton University Press; 1946.

[12] Kendall MG, Stuart A. The advanced theory of statistics, vol. I. London: Charles Griffin \& Co. Ltd.; 1958
[13] Blinnikov S, Moessner R. Expansions for nearly Gaussian distributions. Astron Astrophys Suppl Ser 1998;130:193-205.

[14] Cornish EA, Fisher RA. Moments and cumulants in the specification of distributions. Revue de l'Institut Internat de Statis 1937;4:307-20.

[15] S.R. Jaschke. The Cornish-Fisher-expansion in the context of delta-gammanormal approximations. http://www.jaschke-net.de/papers/CoFi.pdf. Discussion Paper 54, Sonderforschungsbereich 373, Humboldt-Universität zu Berlin; 2001.

[16] IEEE APM Subcommittee. The IEEE reliability test system. IEEE Trans Power Syst 1999;14(3):1010-18. 